\title{
INVESTIGATION OF THE STRAINS AT ZnSe/GaAs INTERFACES BY RAMAN SCATTERING *
}

\author{
W. BALA
}

Institute of Physics, N. Copernicus University, Grudziądzka 5/7, 87-100 Toruń, Poland

\section{Drozdowsiri, AND M. KozIELsKi}

Institute of Physics, Poznan Technical University, Piotrowo 3, 60-965 Poznań, Poland (Received March 21, 1991: in final form July 2, 1991)

\begin{abstract}
Investigations of the strains at the $n$-ZnSe epilayers grown on GaAs substrate using polarized Raman spectra are presented. It has been shown that Raman scattering experiment can be used as a method for investigation of the splitting between the heavy- and light-hole bands in $n$-ZnSe thin films.
\end{abstract}

PACS numbers: 78.30. Hv, 61.70. Wp

\section{Introduction}

There is a renewed interest in the basic properties and potential applications of semiconductor heterostructures made of III-V and II-VI compound semiconductor materials [1-3]. This interest stems, in part, from the advances in epitaxial-growth techniques, which have yielded the capability of obtaining high-quality II-VI layers on III-V substrates with abrupt interfaces on atomic scales. The $\mathrm{ZnSe} / \mathrm{GaAs}$ system is one of such II-VI/III-V systems that have recently attracted considerable attention [1-20]. GaAs is extensively used as a substrate material, because it crystalizes in the same crystal structure as $\mathrm{ZnSe}$ and high-quality and large-area wafers are available. However, there are misfits in lattice constants and thermal expansion coefficients between these two materials. The misfits cause the introduction of elastic strain and/or misfit dislocations in $\mathrm{ZnSe}$ epitaxial layers and have considerable effects on the band-structural, electrical and optical properties of $\mathrm{ZnSe}$ epitaxial layers [21-23]. The quite small lattice mismatch

*This work was supported by the Project CPBP 01.08. 
of only $0.27 \%$ at room temperature when $\mathrm{ZnSe}$ is grown on a $\mathrm{GaAs}$ substrate is attractive for making use of $\mathrm{ZnSe} / \mathrm{GaAs}$ structure and quantum wells.

In this paper we present investigations of the strains at $\mathrm{ZnSe} / \mathrm{GaAs}$ interface by Raman scattering method. The results of these investigations bring new experimental elements to the active field of interfaces and heteroepitaxy between II-VI and III-V compound semiconductors. For many of such systems one has to deal with lattice mismatch between the constituent materials which immediately raises the question about the role of internal strains versus epilayer thickness in the determination of heterojunction properties. This paper shows that Raman scattering can be a valuable tool in addressing this issue and can complement very effectively conventional techniques for interface characterization.

\section{Description of the strains at the ZnSe-substrate interface}

Figure 1 shows band structures of $\mathrm{ZnSe}$ at $\Gamma$ point under unstrained and strained conditions. The valence band at the $\Gamma$ point of the unstrained $\mathrm{ZnSe}$ layer

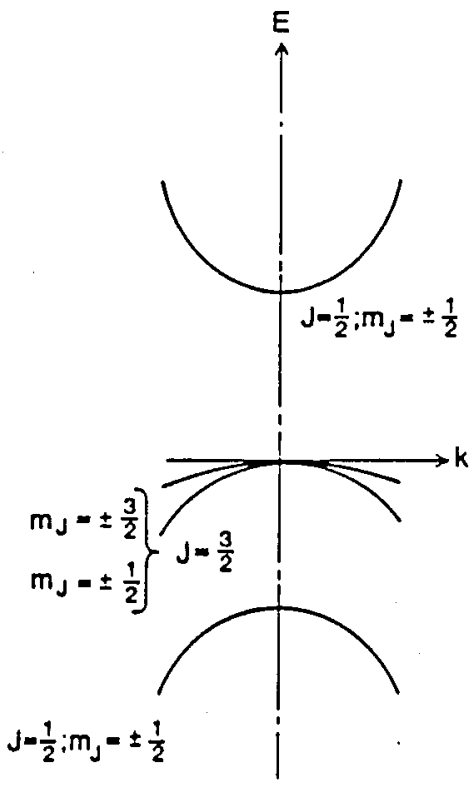

(a)

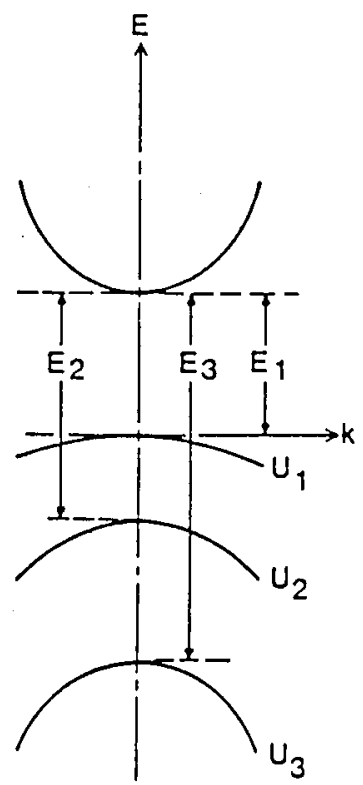

(b)

Fig. 1. Schematic representation of $\mathrm{ZnSe}$ (a) unstrained band structure in $T_{d}$ symmetry and (b) strained due to biaxial compressive stress band structure in $D_{2 d}$ symmetry [8].

consists of a fourfold degenerate $P_{3 / 2}$.valence band $\left(J=3 / 2, m_{J}= \pm 3 / 2, \pm 1 / 2\right.$ ), separated by an energy $\Delta_{0}$ from the twofold degenerate $P_{1 / 2}\left(J=1 / 2, m_{J}=\right.$ $\pm 1 / 2$ ) lower-lying valence band as a result of spin-orbit splitting. Under biaxial compressive stress, the uppermost valence band is split into a light-hole $U_{1}$ branch 
$\left(J=3 / 2, m_{J}= \pm 1 / 2\right)$ and a heavy-hole $U_{2}$ branch $\left(J=3 / 2, m_{J}= \pm 3 / 2\right)[5$, $24,25]$. In addition, the hydrostatic component of the stress shifts the center of gravity of this pair and the spin-orbit split-off band $U_{3}$, relative to the conduction band. For $k=0$ the energy shifts of the three bands with respect to the conduction band can be calculated $[4,26,27]$ as:

$$
\begin{aligned}
& \Delta E_{1,2}=E_{\mathrm{H}}+E_{\mathrm{U}}, \\
& \Delta E_{3}=-E_{\mathrm{H}}, \\
& E_{\mathrm{H}}=\left[2 a\left(C_{11}-C_{12}\right) / C_{11}\right] \varepsilon, \\
& E_{\mathrm{U}}=\left[-b\left(C_{11}+2 C_{12}\right) / C_{11}\right] \varepsilon .
\end{aligned}
$$

Here $C_{i j}$ are the elastic stiffness coefficients, $a$ is the hydrostatic deformation potential, $b$ is the shear deformation potential and $\varepsilon$ is the magnitude of the strain tensor components. The $\varepsilon$ is defined to be positive for compressive strain. The equations above show features in band gap shifts. The hydrostatic part of in-plane compressive strain increases the energy distance of all three bands to the conduction band (for $a$ being negative), whereas the shear deformation part pushes, for negative $b$, the light-hole band $\left(m_{J}= \pm 1 / 2\right)$ towards lower energies and heavy-hole band $\left(m_{J}= \pm 3 / 2\right)$ towards higher energies with respect to the center of gravity of the $J=3 / 2$ valence bands levels. For the biaxial stress parallel to the heterointerface, non-zero components of strain tensor are $[24,28]$ :

$$
\begin{aligned}
& \varepsilon=-\varepsilon_{x x}=-\varepsilon_{y y}=\left(C_{11} / 2 C_{12}\right) \varepsilon_{z z}, \\
& \varepsilon_{z z}=\left(a_{\perp}-a_{\mathrm{ZnSe}}\right) / a_{\mathrm{ZnSe}}, \\
& a_{\perp}=a_{\mathrm{GaAs}}+\left(a_{\mathrm{ZnSe}}-a_{\mathrm{GaAs}}\right)\left(C_{11}+2 C_{12}\right) / C_{11},
\end{aligned}
$$

where $a_{\perp}$ is the lattice constant of thin $\mathrm{ZnSe}$ layer on the GaAs substrate, $a_{\mathrm{GaAs}}$ and $a_{\mathrm{ZnSe}}$ are the bulk unstrained lattice constants of $\mathrm{GaAs}$ and $\mathrm{ZnSe}$, respectively, and $C_{i j}$ are the elastic constants.

\section{Determination of the strains at the ZnSe-substrate interface by luminescence and Raman scattering}

Figure 2 shows the dependence of the $\Delta E$ values, obtained from Eqs. (1)-(3), for increasing $\varepsilon$. The experimental data points were obtained from the energy positions of the free exciton luminescence $E_{x}$ and the most prominent bound-exciton line $I_{2}$ (called $I_{x}$ therein) in $\mathrm{ZnSe}$ films of different thicknesses $[4,9]$.

As we noted above, the lattice constants of $\mathrm{ZnSe}(0.5669 \mathrm{~nm})[29]$ is slightly larger than that of GaAs $(0.5654 \mathrm{~nm})$ [30]. Hence the epitaxial growth of ZnSe on $\mathrm{GaAs}$ surface will produce a biaxial compression of the unit cell of $\mathrm{ZnSe}$ in the 


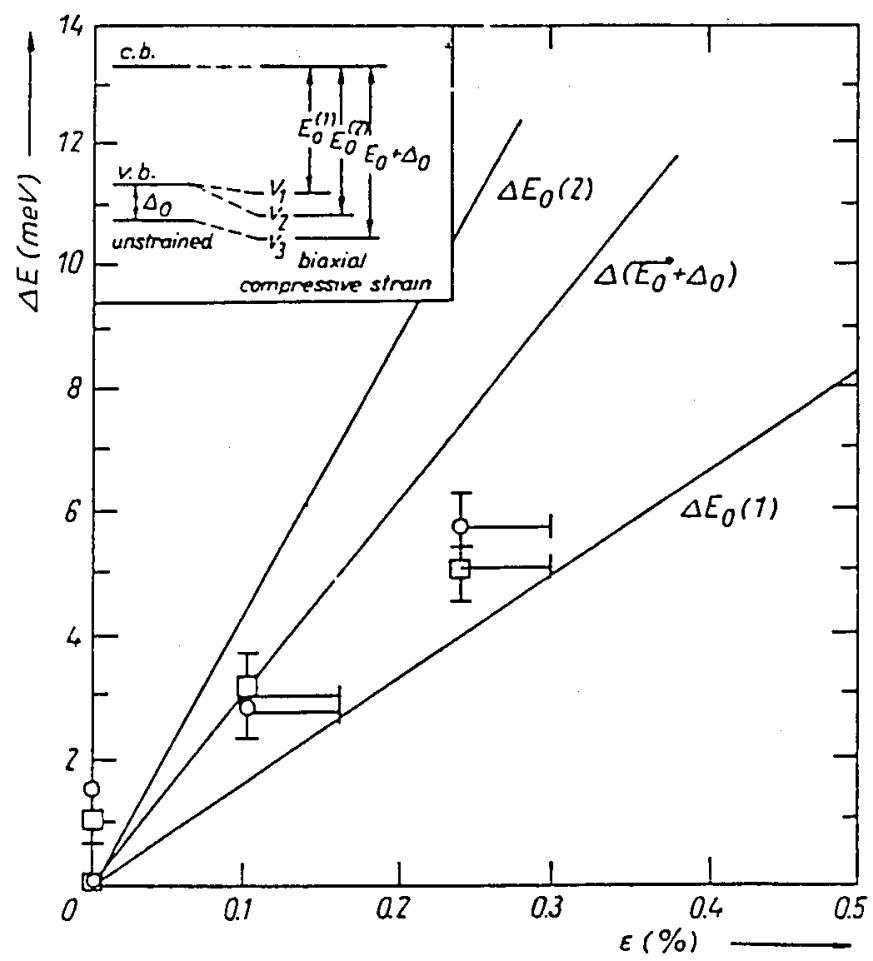

Fig. 2. Variation of the conduction band to valence band transition energies as a function of the biaxial compressive strain. Data points are the shifts in the photoluminescence peak energies observed in the experiment $[4,9]$. The insert defines the transitions discussed in the text.

grown surface. Under these conditions the LO phonon frequency $\omega$ depends on $\varepsilon$ according to the following expression [7, 31-33]:

$$
\Delta \omega_{\mathrm{LO}}=\omega-\omega_{0}=2 \Delta \Omega_{\mathrm{II}}-(2 / 3) \Delta \Omega,
$$

where $\omega_{0}$ is the zero strain frequency of LO phonon and $\Delta \Omega_{\mathrm{H}}$ and $\Delta \Omega$ are given respectively by

$$
\begin{aligned}
& \Delta \Omega_{\mathrm{H}}=\left[(p+2 q) / 6 \omega_{0}^{2}\right]\left[\left(S_{11}+2 S_{12}\right) /\left(S_{11}+S_{12}\right)\right] \omega_{0} \varepsilon, \\
& \left.\Delta \Omega=\left[(p-q) / 2 \omega_{0}^{2}\right]\left[S_{11}-S_{12}\right) /\left(S_{11}+S_{12}\right)\right] \omega_{0} \varepsilon
\end{aligned}
$$

with $p$ and $q$ being the splitting parameters, $S_{i j}$ - the elastic compliance constants. Quantitative estimates of the elastic strains $\varepsilon$ in $\mathrm{ZnSe}$ layers of $\mathrm{ZnSe} / \mathrm{GaAs}$ heterostructures as a function of layer thickness obtained by Olego et al. [7] from Raman scattering measurements are plotted in Fig. 3. Similar results obtained from photoluminescence (PL), X-ray diffraction (XRD), and transmission electron microscopy (TEM) are also included for comparison [36]. 


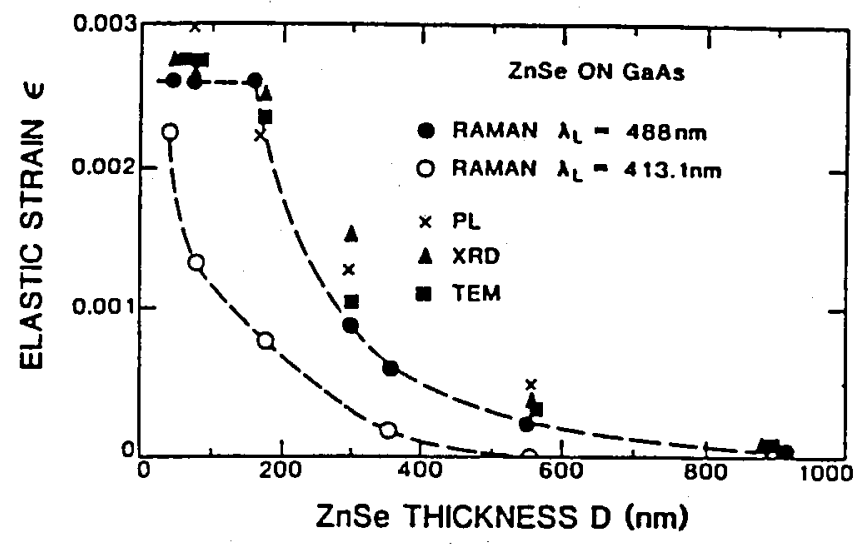

Fig. 3. Dependence of the elastic strains in the $\mathrm{ZnSe}$ layers of $\mathrm{ZnSe} / \mathrm{GaAs}$ heterostructures as a function of layer thickness obtained by different methods [7].

Kumazaki et al. [2] estimated a term of native lattice deffects by measuring the full width at half maximum $\Gamma$ (FWHM) of Raman spectra. According to this model the variation of LO phonon on the surface reflects a variation of the strain and a number of hillocks of a few $\mu \mathrm{m}$ diameter on surface [22, 23]. Considering the effects of imperfections due to native defects and of elastic and thermal strains in the FWHM they propose a new definition of FWHM as a characterization of crystallinity as follows:

$$
\hbar \Gamma=\hbar \Gamma_{0}+A \exp \left(-\hbar \omega_{0} / k T\right)
$$

where $A$ is a constant, $\omega_{0}-$ the LO phonon frequency, and $k$ - the Boltzmann constant. The first term corresponds to FWHM due to imperfections including both strains without temperature dependence and the second is the phonon one with temperature dependence to the population number of phonons. Fig. 4 shows the FWHM of MBE-ZnSe for two different thicknesses as a function of temperature obtained from Raman scattering measurements [2].

\section{Determination of the strains at the $\mathrm{ZnSe-substrate} \mathrm{interface} \mathrm{using}$ the Raman resonant profiles}

ZnSe epilayers used in experiment were deposited on (001) GaAs substrates by MBE method $[34,35]$. The Raman scattering spectra of $\mathrm{ZnSe}$ epilayer were measured in the temperature range between $300 \mathrm{~K}$ and $500 \mathrm{~K}$ using the experimental technique described elsewhere [17-19]. At room temperature the energy band gap $E_{\mathrm{g}}$ for $\mathrm{ZnSe}$ is larger than energy of incident photon. With increasing temperature $E_{\mathrm{g}}$ decreases, and the intensity of the $\mathrm{LO}_{\mathrm{ZnSe}}$ phonon line increases. When energy of the excitation line $E_{\text {ex }}$ is equal to $E_{\mathrm{g}}$ resonance occurs and we observe the maximum of the Raman resonant profile for $\mathrm{LO}_{\mathrm{ZnSe}}$ line. For the $\mathrm{ZnSe}$ 


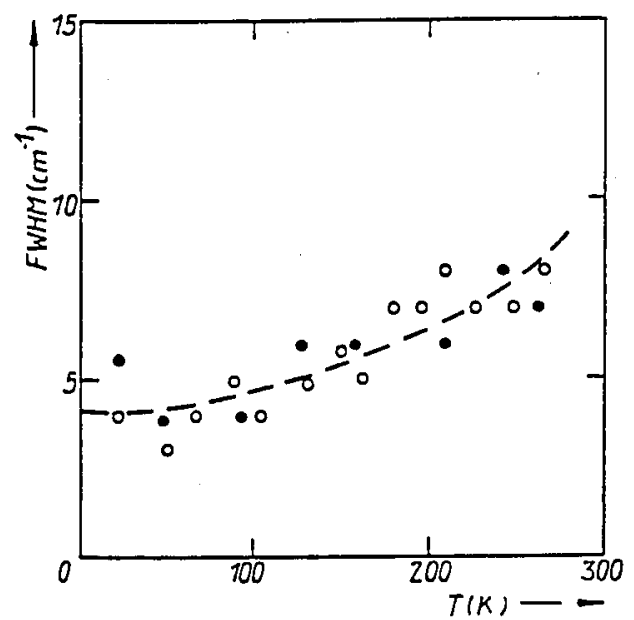

Fig. 4. Full width at half maximum (FWHM) of $\mathrm{ZnSe}$ epilayer on GaAs with $0.45 \mu \mathrm{m}$ (open circles) and $1.2 \mu \mathrm{m}$ (full circles) as a function of temperature [2].

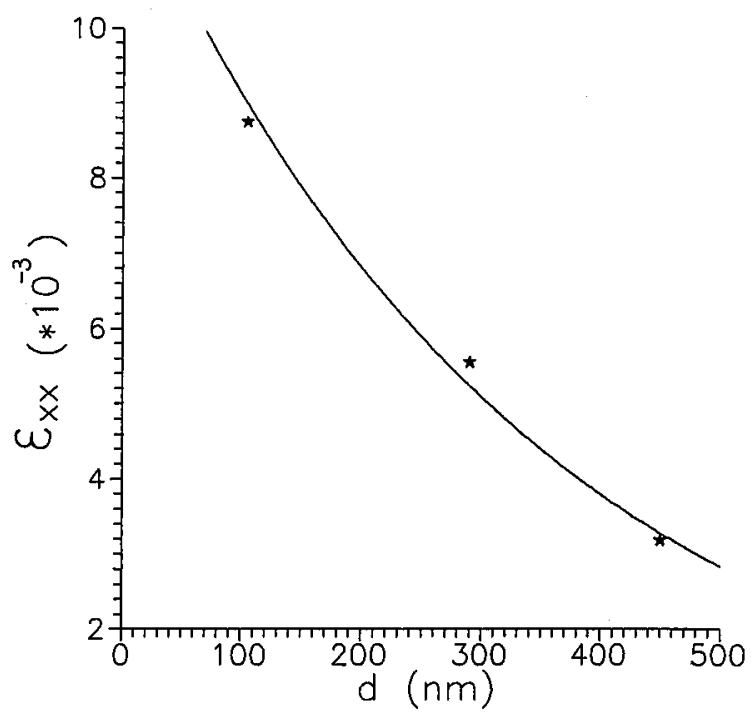

Fig. 5. Dependance of the elastic strain in $\mathrm{ZnSe}$ epilayers on $\mathrm{GaAs}$ as a function of layer thickness obtained by Raman profiles method [18,19].

epilayers with different thickness the maximum of the Raman resonant profile occurs at different temperatures. When the thickness of the layer, $d$, increases the maximum of Raman resonant profile shifts to lower temperature [19]. From the difference between the excitation energy and the energy band gap $\left(E_{\mathrm{ex}}-E_{\mathrm{g}}=E_{\mathrm{U}}\right)$, one can calculate the internal strain using Eq. (3b). 


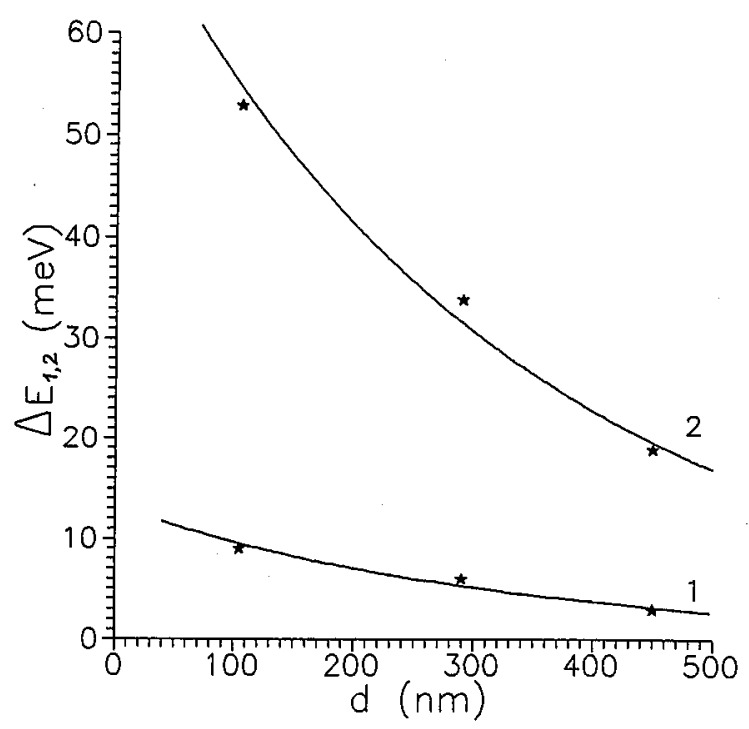

Fig. 6. The energy shifts of the band gaps between conduction and two strain-split valence bands of $\mathrm{ZnSe}$ epilayer on $\mathrm{GaAs}$ as a function of layer thickness.

Fig. 5 shows the elastic strain $\varepsilon_{x x}$ as a function of $\mathrm{ZnSe}$ epilayer thickness. As it is seen from Fig. 5 the value of the elastic strain decreases when the thickness of the epilayer increases. However, the values of $\varepsilon_{x x}$ obtained by Raman resonant profiles are larger than these estimated by another methods (see Fig. 3).

From the known values of $\varepsilon$ one can also estimate the energy shifts $\Delta E_{1,2}$ using Eqs. (1)-(3). The variation in the energy gaps with increasing strain for $\mathrm{ZnSe}$ epilayers on GaAs, obtained from Raman resonant profiles are plotted in Fig. 6. As it can be seen from Fig. 6, the values obtained by this method are in good agreement with the data obtained from photoluminescence measurements (see Fig. 2).

\section{References}

[1] J. Gutowski, N. Presser, G. Kudlek, Phys. Status Solidi A 120, 11 (1990).

[2] K. Kumazaki, K. Imai, A. Odajima, Phys. Status Solidi A 119, 117 (1990).

[3] D.J. Olego, Phys. Rev. B 30, 12743 (1989).

[4] J.E. Potts, H. Cheng, S. Mohapatra, T.L. Smith, J. Appl. Phys. 61, 333 (1986).

[5] K. Ohkawa, T. Mitsuya, O. Yamazaki, Phys. Rev. B 38, 12465 (1987).

[6] T. Matsumoto, S. Yoshida, T. Ishida, Jpn. J. Appl. Phys. 25, L413 (1986).

[7] D.J. Olego, K. Shahzad, J. Petruzzello, D. Cammack, Phys. Rev. B 36, 7674 (1987).

[8] K. Shahzad, D.J. Olego, C.G. Van de Walle, Phys. Rev. B 38, 1417 (1988). 
[9] K. Shahzad, Phys. Rev. B 38, 8309 (1988).

[10] N. Shibata, A. Ohki, S. Zembutsu, J. Cryst. Growth 86, 268 (1988).

[11] N. Shibata, S. Zembutsu, T. Miyashita, Jpn. J. Appl. Phys. 25, L335 (1986).

[12] N. Itoh, K. Okamoto, H. Ogawa, T. Kawabata, S. Koike, Appl. Surf. Sci. 33/34, 413 (1988).

[13] N. Matsumura, M. Tsubokura, J. Saraie, J. Cryst. Growth 95, 525 (1989).

[14] B.J. Skromme, M.C. Tamargo, J.L. de Miguel, R.E. Nahory, Appl. Phys. Lett. 53, 2217 (1988).

[15] S. Colak, T. Marshall, D. Cammack, Solid-State Electron. 32, 647 (1989).

[16] S. Lee, B. Hillebrands, G.J. Stegeman, H. Cheng, J.E. Potts, F. Nizzoli, J. Appl. Phys. 63, 1914 (1988).

[17] W. Bała, M. Drozdowski, M. Kozielski, Acta Phys. Pol. A79, 225 (1991).

[18] W. Bala, M. Drozdowski, M. Kozielski, in: Proc. 4th Conf. Surface Physics, Eódź 1989, ed. Eódź University, Vol. VII, Eódź 1990, p. 31.

[19] W. Bala, M. Drozdowski, M. Kozielski, in: Proc. 5th Conf. Surface Physics, Eódź 1990, ed. Łódź University, Vol. IX, Łódź 1991, p. 35.

[20] D. Walsh, K. Mazuruk, M. Benzaquen, Phys. Rev. B 36, 2883 (1987).

[21]. T. Yao, Jpn. J. Appl. Phys. 25, L544 (1986).

[22] T. Matsumoto, T. Kato, M. Hosoki, T. Ishida, Jpn. J. Appl. Phys. 26, L576 (1987).

[23] S. Nakashima, A. Fujii, K. Mizoguchi, A. Mitsuishi, K. Yoneda, Jpn. J. Appl. Phys. 27, 1327 (1988).

[24] H. Asai, K. Oe, J. Appl. Phys. 54, 2052 (1983).

[25] J. Dean, Phys. Status Solidi A 81, 625 (1984).

[26] Y.R. Lee, A.K. Ramdas, L.A. Kołodziejski, R.L. Gunshor, Phys. Rev. B 38, 13143 (1988).

[27] G.E. Pikus, G.L. Bir, Sov. Phys.-Solid State 1, 136 (1959); 1, 1502 (1960).

[28] G.H. Olsen, C.J. Nuese, R.T. Smith, J. Appl. Phys. 49, 5523 (1978).

[29] R. Feder, T. Light, J. Appl. Phys. 39, 4870 (1968).

[30] H.P. Singh, B. Dayal, Phys. Slatus Solidi 23, K93 (1967).

[31] F. Cerdeira, C.J. Buchenauer, F.H. Pollak, M. Cardona, Phys. Rev. B 5, 580 (1972).

[32] B. Jusserand, P. Voissin, M. Voos, L.L. Chang, E.E. Mendez, L. Esaki, Appl. Phys. Lett. 46, 678 (1985).

[33] L.H. Shon, K. Inoue, O. Madsuda, K. Murase, T. Yokogawa, M. Ogura, Solid State Commun. 67, 779 (1988).

[34] M. Kozielski, M. Drozdowski, W. Bala, F. Firszt, Acta Phys. Pol. A73, 369 (1988).

[35] W. Bała, F. Firszt, M. Grinberg, M. Drozdowski, M. Kozielski, Acta Phys. Pol. A75, 75 (1989).

[36] K. Mohammed, D.A. Cammack, R. Dalby, P. Newbury, B.L. Greenberg, J. Petruzzello, R. Bhargava, Appl. Phys. Lett. 50, 37 (1987). 